\title{
Sulfoquinovosyldiglyceride and Triglycosyldiglyceride in Rice Grains
}

\author{
Yasuo Kondo, Seisuke ITo and Yasuhiko FuJINo \\ Department of Agricultural Chemistry, Obihiro Chikusan University, \\ Obihiro, Hokkaido, Japan
}

Received August 13, 1974

\begin{abstract}
Sulfoquinovosyldiglyceride and triglycosyldiglyceride were isolated from rice grains, mainly by column chromatography and thin-layer chromatography. The major component fatty acids were palmitic, oleic, stearic and linoleic acids in sulfoquinovosyldiglyceride whereas they were linoleic, palmitic and oleic acids in triglycosyldiglyceride, in decreasing order of amount. The component sugar in sulfoquinovosyldiglyceride was sulfoquinovose, while those in triglycosyldiglyceride were galactose and glucose, the latter being predominant.
\end{abstract}

Monoglycosyldiglyceride (MGD), diglycosyldiglyceride (DGD), sulfoquinovosyldiglyceride (SQD) and triglycosyldiglyceride (TGD) are representative glyceroglycolipids in the plant kingdom. We recently reported the existence of MGD and DGD in rice grains. ${ }^{11}$ The present paper describes the isolation and chemical characterization of SQD and TGD from rice grains.

\section{METHODS}

Extraction and fractionation of lipid. Equal amounts of brown rice grains of Ishikari-hakumo, Horyu and Koshijiwase species were mixed together, smashed to powder of 30 mesh and extracted with chloroformmethanol $(2: 1, \mathrm{v} / \mathrm{v})$ to collect total lipid. ${ }^{2}$ The yield was $2.5 \%$ of the sample grain. The lipid was subjected to silicic acid column chromatography, using sequential elution with chloroform, acetone and methanol.3) Crude neutral lipids were eluted with chloroform, glycolipids with acetone and phospholipids with methanol. The ratio of the three crude fractions was approximately $90: 6: 4$.

Isolation of $S Q D$ and $T G D$. The crude glycolipids were subjected to silicic acid column chromatography using stepwise elution with a solvent system of chloroform-acetone. ${ }^{41}$ A fraction containing SQD and TGD was obtained in the last stage of elution with chloroform-acetone $(0: 10)$. The crude phospholipids, contaminated with some SQD and TGD, were chromatographed on a florisil column with a solvent system of acetone-methanol.5) A fraction including SQD and TGD was eluted with acetone-methanol $(0: 10 \sim 6: 4$, $v / v)$. The two fractions were combined and subjected to preparative thin-layer chromatography with a solvent system of chloroform-methanol-water $(70: 35: 7, \mathrm{v} / \mathrm{v} / \mathrm{v})$. Thus SQD and TGD were prepared separately, and were purified by repeating the chromatography. Yield of each lipid was under $1 \%$ from the crude glycolipids.

Decomposition of $S Q D$ and $T G D$. Each glyceroglycolipid was methanolyzed with $5 \% \mathrm{HCl}$ in methanol at $100^{\circ} \mathrm{C}$ for $4 \mathrm{hr}$. After cooling, fatty acid methylesters were extracted from the reaction mixture with hexane-ether $(1: 1, v / v)$. The residual methanolic phase was passed through an ion exchange column to prepare the water-soluble fraction. On the other hand, TGD was partially hydrolyzed with $0.4 \mathrm{~N} \mathrm{KOH}$ in methanol at $37^{\circ} \mathrm{C}$ for $4 \mathrm{hr}$. Fatty acid was removed with hexane under acidic conditions, and the residual liquid was passed through Dowex $50\left(\mathrm{H}^{+}\right)$and Dowex 2 $\left(\mathrm{OH}^{-}\right)$columns to obtain deacylated TGD (triglycosylglycerol).

Infrared spectrometry. The glyceroglycolipid was pelleted with $\mathrm{KBr}$, and the infrared spectrum was taken on an infrared spectrophotometer (Model IR-G, Japan Spectroscopic Co., Ltd., Tokyo).

Paper chromatography. Sugar constituents of the glyceroglycolipid were analyzed by ascending chromatography on Toyo filter paper No. 51. The solvent system was $n$-butanol-pyridine-water $(6: 4: 3, \mathrm{v} / \mathrm{v} / \mathrm{v})$ for methylsulfoquinovoside and phenol-water $(5: 2$, $w / w)$ for triglycosylglycerol. The detection reagent was $\mathrm{AgNO}_{3}-\mathrm{NaOH}$ solution. ${ }^{6)}$

Gas-liquid chromatography. Gas-liquid chromatography was performed on a gas chromatograph (Type 063, Hitachi Ltd., Tokyo) equipped with a hydrogen flame ionization detector.

Fatty acid methylesters were chromatographed at $180^{\circ} \mathrm{C}$ through a $0.3 \times 200 \mathrm{~cm}$ stainless column packed with $10 \%$ DEGS on Uniport B. Methylglycosides were analyzed as trimethylsilyl ether derivatives ${ }^{7}$ at 
$170^{\circ} \mathrm{C}$ on a $0.3 \times 200 \mathrm{~cm}$ glass column packed with $5 \%$ SE-30 on Chromosorb W. Peaks in the chart were identified by cochromatography with commercial standard preparations or by comparison of the relative retention times with those of standard specimens.

\section{RESULTS AND DISCUSSION}

Thin-layer chromatogram

A thin-layer chromatogram of SQD and

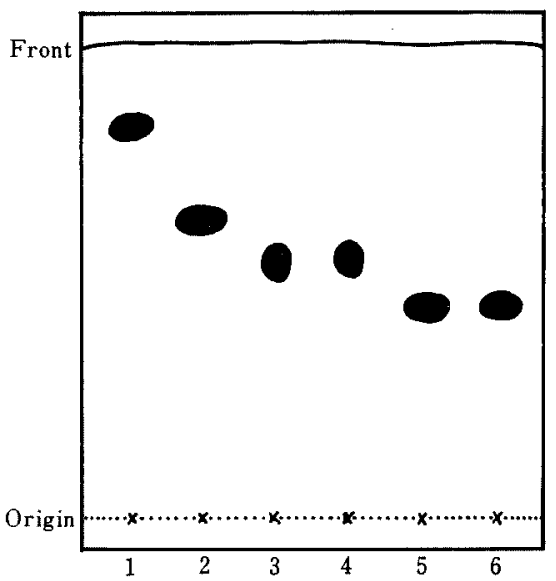

FIG. 1. Thin-layer Chromatogram of SQD and TGD Isolated from Rice Grains.

1. Monogalactosyldiglyceride, standard. 2. Digalactosyldiglyceride, standard. 3. Sulfoquinovosyldiglyceride, standard. 4. Sulfoquinovosyldiglyceride, sample. 5. Trigalactosyldiglyceride, standard.

Triglycosyldiglyceride, sample.

Development: $\mathrm{CHCl}_{3}-\mathrm{MeOH}-W a t e r(65: 25: 4 \mathrm{v} / \mathrm{v} / \mathrm{v})$

Detection: Anthrone reagent.
Table I. Fatty Acid Compositions OF RICE SQD AND TGD

\begin{tabular}{ccc} 
& \multicolumn{1}{c}{ SQD } & \multicolumn{1}{c}{ TGD } \\
\hline $14: 0$ & $5.7^{\%}$ & $1.4^{\%}$ \\
$16: 0$ & 44.8 & 25.6 \\
$16: 1$ & 3.3 & 1.3 \\
$17: 0$ & - & 2.0 \\
$18: 0$ & 12.7 & 7.5 \\
$18: 1$ & 22.5 & 19.4 \\
$18: 2$ & 10.1 & 40.3 \\
$18: 3$ & 0.9 & 2.5 \\
\hline
\end{tabular}

TGD isolated from rice grains is shown in Fig. 1. Sample lipids gave single spots and the $R f$ values agreed well with those of reference SQD and TGD obtained from pumpkin. ${ }^{8)}$

\section{Infrared spectrum}

The infrared spectrum of rice SQD is shown in Fig. 2. Strong absorption at $1730 \mathrm{~cm}^{-1}$ was due to ester bonds and those at $1170 \mathrm{~cm}^{-1}$ and $1035 \mathrm{~cm}^{-1}$ to $\mathrm{S}-\mathrm{O}$ linkages. The pattern of the spectrum closely coincided with that of algal SQD ${ }^{9}$ or alfalfa SQD. ${ }^{10}$ The spectrum of TGD, though not shown, resembled those of rice MGD and $\mathrm{DGD}^{1)}$ except that in TGD the ratio of absorption at $1070 \mathrm{~cm}^{-1}$ due to $\mathrm{OH}$ groups of sugar moieties to the absorption at $1730 \mathrm{~cm}^{-1}$ due to ester bonds was much higher than in MGD and DGD.

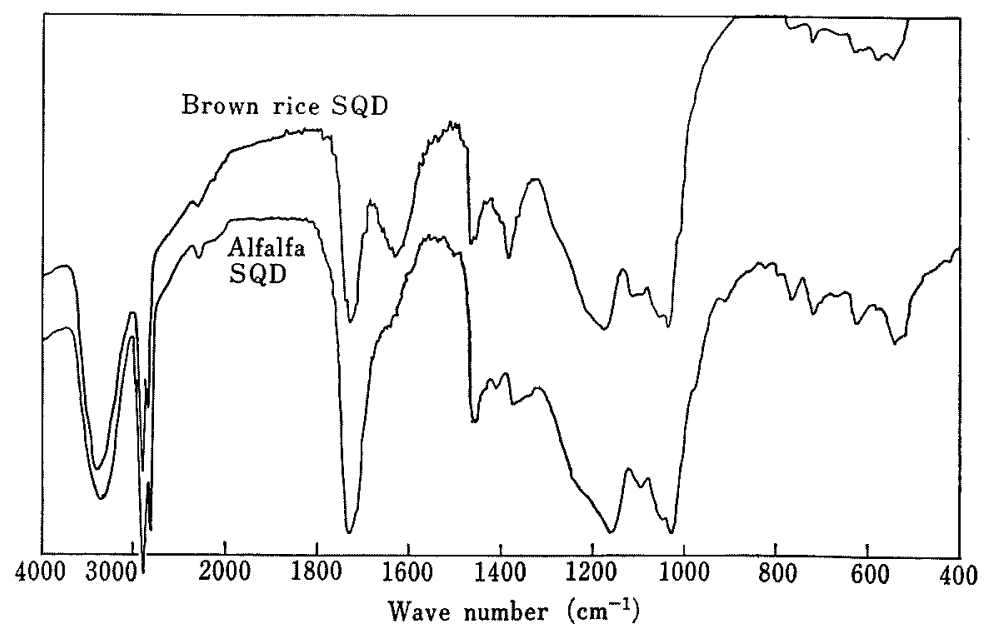

FIG. 2. Infrared Spectrum of Rice SQD. 
Fatty acid composition

The fatty acid composition of rice SQD and TGD is shown in Table $I$. Seven fatty acids were found in SQD. The main ones were palmitic, oleic, stearic and linoleic acids in decreasing amount. The pattern was very similar to that of rice MGD, ${ }^{1}$ implying that both SQD and MGD may be formed in rice from similar species of diglycerides. Eight fatty acids were detected in TGD. The predominant ones were linoleic, palmitic and oleic acids in order of decreasing amount. The profile resembled that of rice $\mathrm{DGD},{ }^{1 /}$ suggesting that TGD might be converted in rice from DGD with a similar type of fatty acid composition.

\section{Sugar composition}

A paper chromatogram of the water-soluble fraction prepared by methanolysis of SQD is shown in Fig. 3. The upper spot corresponded to glycerol, and the lower one corresponded not to methylgalactoside or methylglucoside but to methylsulfoquinovoside derived from alfalfa SQD. ${ }^{10)}$ The results indicate that the sugar component in rice SQD is sulfoquinovose alone.

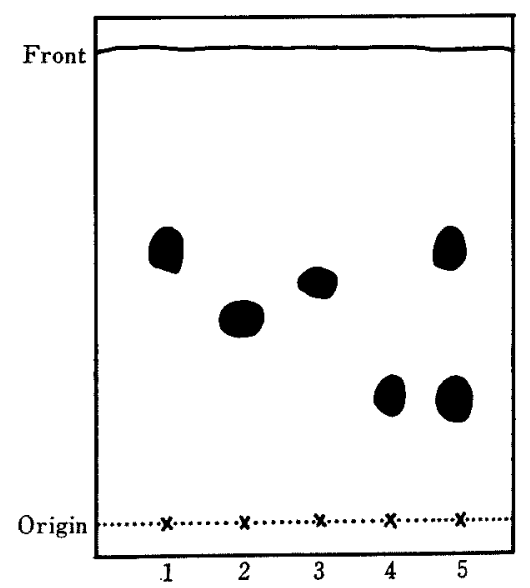

FIG. 3. Paper Chromatogram of Water-soluble Components of Rice SQD.

1. Glycerol, standard. 2. Methylgalactoside, standard. 3. Methylglucoside, standard. 4. Methylsulfoquinovoside, standard. 5. Water-soluble components of rice SQD, sample.

Development : $n$-BuOH-Pyridine-Water $(6: 4: 3, \mathrm{v} / \mathrm{v} / \mathrm{v})$. Detection: $\mathrm{AgNO}_{3}-\mathrm{NaOH}$.

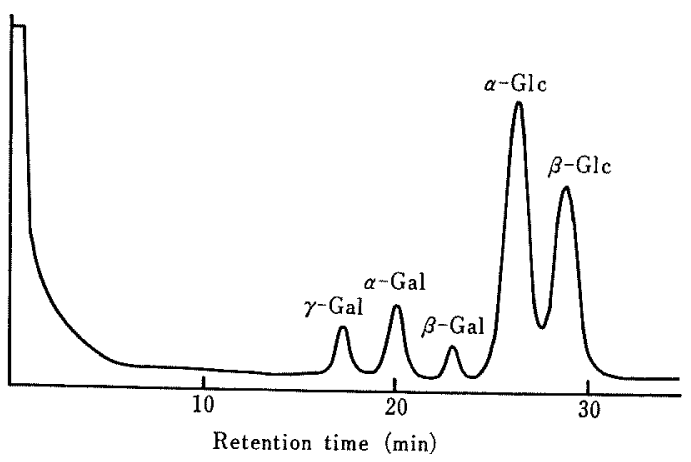

FIG. 4. Gas-liquid Chromatogram of Trimethylsilyl Ether Derivatives of the Water-soluble Components of Rice TGD.

Conditions: see the text.

The $R f$ value on a paper chromatogram of the deacylated TGD agreed with that of triglycosylglycerol prepared from pumpkin TGD ${ }^{8)}$ A gas-liquid chromatogram of the water-soluble fraction obtained by methanolysis of TGD is shown in Fig. 4. Peaks for galactose and glucose isomers were detected, although the glycerol peak seemed to be concealed in the initial peak of the solvent system under the conditions used in this experiment. The approximate ratio of galactose to glucose was $18: 82$. This finding, together with the data that rice MGD contained only galactose and rice DGD contained galactose and glucose in a ratio of $6: 1,{ }^{1}$ suggests that the proportion of galactose to glucose tends to decrease gradually as the number of component sugars increases in rice glyceroglycolipids. The position and mode of combination of the three hexose constituents still remain to be elucidated in rice $\mathrm{TGD}$.

\section{REFERENCES}

1) Y. Fujino and S. Sakata, Cereal Chem., 50, 379 (1973).

2) J. Folch, M. Lees and G. H. Sloane-Stanley, $J$. Biol. Chem., 226, 497 (1957).

3) G. Rouser, G. Kritchevsky, G. Simon and G. T. Nelson, Lipids, 2, 37 (1967).

4) M. L. Vorbeck and G. V. Marinetti, J. Lipid Res., 6, 3 (1965).

5) J. S. O'Brien and A. A. Benson, ibid., 5, 432 (1964)

6) W. E. Trevelyan, D. P. Procter and J. S. Harrison, 
Nature, 166, 444 (1950).

7) H. E. Carter and R. C. Gaver, J. Lipid Res., 5, 126 (1956).

8) S. Ito and Y. Fujino, Nippon Nōgeikagaku Kaishi,
48, 431 (1974).

9) A. Radunz, Z. Physiol. Chem., 350, 411 (1969).

10) S. Ito and Y. Fujino, Nippon Nögeikagaku Kaishi, 46, 319 (1972). 